\title{
Preliminary study on the working characteristics of thermoelectric power generation
}

\author{
Mu Wang \\ North China Electric Power University (Baoding), Baoding. 071000, China \\ 2475593950@qq.com
}

\begin{abstract}
Keywords: Thermoelectric power generation, performance characteristics, Experimental analysis, Prospect.
\end{abstract}

\begin{abstract}
China is the world's largest coal production and consumption country, coal accounted for $76 \%$ of energy consumption of goods, which is also the main source of air pollution in China. Therefore, the development and utilization of renewable energy, improvement of the existing energy structure and active search for new energy saving technology is imminent. This paper discusses the application status of thermoelectric power generation, the influence of hot and cold side, cooling components on its properties and affirmed the application prospect of thermoelectric power generation.
\end{abstract}

\section{Introduction}

Thermoelectric power generation technology has become a strategic technology of energy and environment and has received strong support of all countries in recent years. Study on thermoelectric power generation mainly concentrated on the development of materials for high merit, design and optimization of module and system for materials in the high quality and so on. Although the thermoelectric refrigeration technology has been developing rapidly in the past 50 years, but there are still disadvantages such as low refrigeration coefficient (COP), high cost per refrigeration, especially less economic competitiveness with traditional compression refrigeration in high power applications So the research on improving the thermoelectric refrigeration coefficient, further broadening its application scope and breadth has important significance.

\section{The Basic Principle}

Thermoelectric power generation is based the thermoelectric effect, with energy transported between thermal energy and electric energy by carrier. Thermoelectric power generation consists of five different effects: the Seebeck effect, Parr effect, Thomson effect, Joule effect and Fourier effect. The first three are reversible effects of electricity and heat conversion, the latter two are hot irreversible effects. The effects of the five constitute the theoretical foundation of thermoelectric research. The semiconductor thermoelectric generator can be divided into the heat source, the semiconductor thermoelectric device, a heat insulation layer, heat dissipation components and strength components in several parts, in addition to the power regulator and other related components.

\section{Experimental Analysis of Actual Performance}

To simplify the problem, we make the following assumptions in the process of modeling:

1. There is no difference of the 9 thermoelectric power generation chips, that is to say the thermal conductivity, length, cross-sectional area and any other parameters are all same.

2. Ignore the thermal radiation of hot and cold junction and the system's heat exchange with the surroundings;

3. The heat conducted by the graphite sheet is uniform in different area of the graphite sheet. 
And the schematic diagram of the temperature difference power generation device just as the Figure 1 shows, the thermoelectric power with variation of temperature difference is shown in Figure 2.

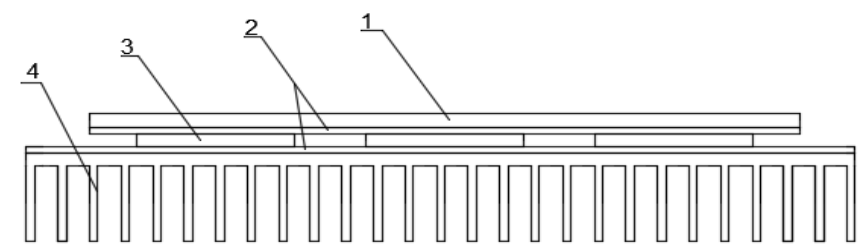

Figure 1.The schematic diagram of the temperature difference power generation device Aluminum sheet; 2-Graphite sheet; 3-Thermoelectric power generation sheet; 4-Air-cooling fin.

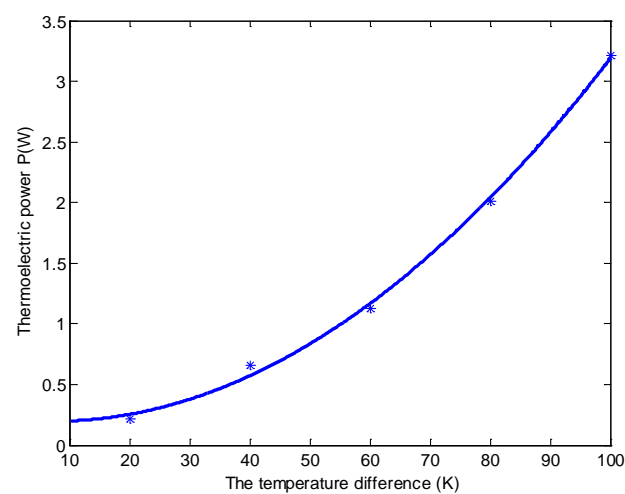

Figure2. The thermoelectric power with variation of temperature difference

In the practical research, we just analysis the steady heat conduction of the device. The beam through the final lens focus on the heat collecting aluminum sheet, and the heat transfer rate of the aluminum sheet is $\phi \square$ which is also the total heat that the device absorbs. One part of the heat flows through the thermoelectric power generation chip, then converses into electrical power, of which the power is $P$ and the conversion efficiency is $\eta_{1}$.The other flows from the cold junction, the air-cooling fin, and lost to the surroundings .We use the letter $\phi_{1}$ to describe the heat transfer rate that is used by thermoelectric power generation chip and $\phi_{2}$ to describe the lost part.

So, the total heat transfer rate conducted by aluminum sheet is:

$$
\phi=\frac{T_{1}-T_{2}}{R_{1}}=\frac{T_{1}-T_{2}}{\frac{\delta_{1}}{A_{1} \lambda_{1}}}
$$

Where $A_{1}$ is the area of the aluminum sheet ; $T_{1}$ is the temperature of the aluminum sheet's upper surface ; $T_{2}$ is the temperature of the aluminum sheet's lower surface ; $R_{1}$ is thermal resistance of the aluminum sheet ; $\delta_{1}$ is the thickness of aluminum sheet ; $\lambda_{1}$ is the thermal conductivity of the aluminum sheet.

The temperature difference between the hot and cold junction of thermoelectric power generation chip:

$\Delta T=T_{3}-T_{4}$

Where $T_{3}$ is the temperature of thermoelectric power generation chip's hot junction; $T_{4}$ is the temperature of thermoelectric power generation chip's cold junction.

We can get the relation between the temperature difference and the rate of power generation is:

$P=f(\Delta T)$

The heat that the thermoelectric power generation sheet consumes to generation electricity is:

$\phi_{1}=\frac{P}{\eta_{1}}$

Look up the thermal physical properties of the dry air at standard atmospheric pressure, and get the Nussle number by the feature correlation equation:

$$
\mathrm{Nu}=\frac{0.4637 \mathrm{Re}^{\frac{1}{2}} \operatorname{Pr}^{\frac{1}{3}}}{\left[1+(0.0207 / \mathrm{Pr})^{\frac{2}{3}}\right]^{\frac{1}{4}}}
$$


So the convection heat transfer coefficient is:

$h_{1}=\frac{\lambda_{2}}{l_{2}} \mathrm{Nu}$

Where $\lambda_{2}$ is the thermal conductivity of air; $l_{2}$ is the length of the fin which is along the direction of the air-flow.

The heat flows lost to the surroundings from the air-cooling fin by convection is:

$\phi_{2}=\eta_{\mathrm{f}} \mathrm{h}_{1} \mathrm{~A}_{3}\left(\mathrm{~T}_{4}-\mathrm{t}_{0}\right)$

Where $\eta_{f}$ is efficiency of the fin; $h_{1}$ is the convective heat transfer coefficient of the fin; $A_{3}$ is the total area of the air-cooling fin that exchanges heat with cold air; $T_{4}$ is the temperature of thermoelectric power generation chip's cold junction; $t_{0}$ is the temperature of the cold air.

To simplify the calculation, we just take the area of the fin into account and ignore the area of the root of the fin. To a certain degree, it is reasonable.

We can get the equation under the law of conservation of energy:

$\phi=\phi_{1}+\phi_{2}$

\section{Outlook and Prospect}

At present, there are mainly two bottlenecks in the development of thermoelectric power generation. For one thing, the thermoelectric conversion efficiency is not high, mainly subject to the performance of semiconductor thermoelectric materials. For another, the application scope is limited. Domestically, the development of thermoelectric power generation technology is slow and the actual application is seldom reported, the typical solar thermoelectric power generation schematic diagram shown in Figure 3.

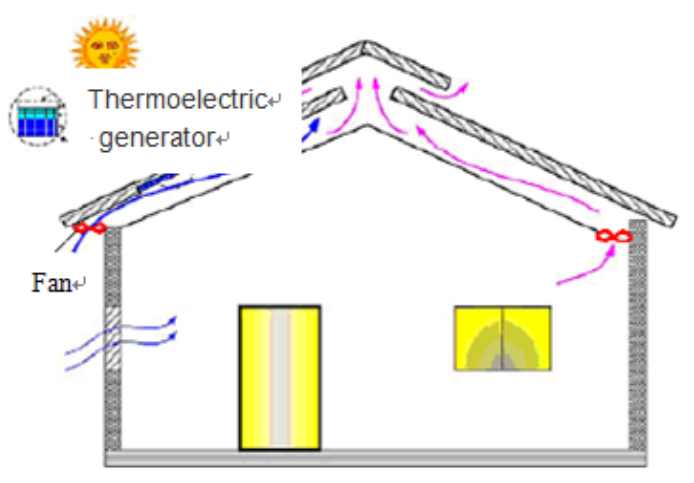

Figure 3 Schematic diagram of a typical solar thermoelectric power generation

\section{Conclusions}

This chapter analyzes the performance characteristics of irreversible semiconductor power generation system and derives some basic relations. Based on the basic relation, we draw a semiconductor generator important optimization performance characteristic curve, obtain the optimal operating parameter, determine the maximum efficiency of the generator working conditions and optimize various important parameters in a given heating rate. The results have guiding significance to optimize the design and manufacture of actual semiconductor power generation system.

\section{References}

[1]Reng Depeng, Jia Yang, Numerical simulation for operating characteristics of thermogenerator, Spacecraft Engineering, 2008, 17(4):56-61(in Chinese).

[2]Xuan X C, Li D. Optimization of a combined thermionic-thermoelectric generator, Journal of Power Sources, 2003, 115:167-170

[3]Yang Yongjie, Zhang Yusheng, Design of a PM 2.5 detecting sensor, Transducer and microsystem, 2014, 33(3). 